\title{
GLIA
}

\section{Making waves for myelin}

DOI:

10.1038/nrn1961

URLs
The outgrowth of oligodendrocyte cell processes is vital for the formation of myelin, but the underlying mechanisms are not well understood. Kim, DiBernardo and colleagues now take us a step closer to understanding these mechanisms with the discovery that a WAVE (Wiskott-Aldrich syndrome protein family verprolin homologous) protein is essential for the morphological development of oligodendrocytes and consequent myelin formation through its influence on cytoskeleton dynamics.

WAVE proteins are widely expressed in the nervous system, but little is known about their functions, particularly in glia. One clear role for these proteins in neurons, however, is that they are involved in regulating the organization of the actin cytoskeleton - which is also a crucial step in the generation of myelin sheaths.

These researchers found that the WAVE1 isoform, but not WAVE2 or WAVE3, is dynamically expressed in oligodendrocytes during development. Moreover, WAVE1 expression coincided with the onset of myelination, and was localized to the leading edge of lamellipodia - actin cytoskeleton projections that reside on migrating cells. The team therefore investigated the function of WAVE1 in oligodendrocytes.

Preventing WAVE1 function disrupted process extension in cultured oligodendrocytes and in oligodendrocytes from WAVE1-knockout mice. However, although this isoform is also expressed in neurons, their development was unaffected. Furthermore, in WAVE1-knockout mice, formation of nodes of Ranvier was compromised, suggesting that WAVE1 is crucial for normal myelination.

These abnormalities in oligodendrocyte development had a profound effect on the overall anatomy of the brain in WAVE1knockout mice. There was a general reduction in brain size, with volume loss being most pronounced in the forebrain and corpus callosum, whereas the lateral ventricles were significantly larger than normal. These findings were associated with an overall reduction in the number of oligodendrocytes participating in myelination, particularly in the corpus callosum.

This elegant study not only sheds light on the mechanisms of

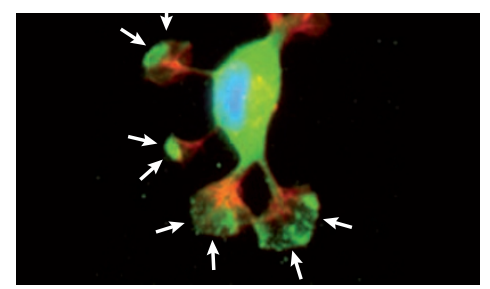

Fluorescence image of WAVE1 localization in lamellipodia of an early-stage oligodendrocyte purified from neonatal rat forebrain. WAVE1 (green) staining localizes to lamellipodia (arrows) of an oligodendrocyte. Red staining represents F-actin. Image courtesy of T. Vartanian and J. A. Sloane, Harvard Medical School, Massachusetts, USA.

myelin formation, but also reveals an important role for WAVE1 in glia. It will be interesting to determine whether WAVE1 contributes to oligodendrocyte maturation as well as the development of myelin.

Alison Rowan

ORIGINAL RESEARCH PAPER Kim, H.--., DiBernardo, A. B. et al. WAVE1 is required for oligodendrocyte morphogenesis and normal CNS myelination. J. Neurosci. 26, 5849-5859 (2006) FURTHER READING Sherman, D. L. \& Brophy, P. J. Mechanisms of axon ensheathment and myelin growth. Nature Rev. Neurosci. 6 , 683-690 (2005) 